\title{
THE SEQUENTIAL APPROACH TO THE PRODUCT OF DISTRIBUTION
}

\author{
C. K. LI
}

(Received 12 June 2000)

\begin{abstract}
It is well known that the sequential approach is one of the main tools of dealing with product, power, and convolution of distribution (cf. Chen (1981), Colombeau (1985), Jones (1973), and Rosinger (1987)). Antosik, Mikusiński, and Sikorski in 1972 introduced a definition for a product of distributions using a delta sequence. However, $\delta^{2}$ as a product of $\delta$ with itself was shown not to exist (see Antosik, Mikusiński, and Sikorski (1973)). Later, Koh and Li (1992) chose a fixed $\delta$-sequence without compact support and used the concept of neutrix limit of van der Corput to define $\delta^{k}$ and $\left(\delta^{\prime}\right)^{k}$ for some values of $k$. To extend such an approach from one-dimensional space to $m$-dimensional, Li and Fisher (1990) constructed a delta sequence, which is infinitely differentiable with respect to $x_{1}, x_{2}, \ldots, x_{m}$ and $r$, to deduce a non-commutative neutrix product of $r^{-k}$ and $\triangle \delta$. Li (1999) also provided a modified $\delta$-sequence and defined a new distribution $\left(d^{k} / d r^{k}\right) \delta(x)$, which is used to compute the more general product of $r^{-k}$ and $\Delta^{l} \delta$, where $l \geq 1$, by applying the normalization procedure due to Gel'fand and Shilov (1964). We begin this paper by distributionally normalizing $\Delta r^{-k}$ with the help of distribution $x_{+}^{-n}$. Then we utilize several nice properties of the $\delta$-sequence by Li and Fisher (1990) and an identity of $\delta$ distribution to derive the product $\Delta r^{-k} \cdot \delta$ based on the results obtained by Li (2000), and $\mathrm{Li}$ and Fisher (1990).
\end{abstract}

2000 Mathematics Subject Classification. 46F10.

1. The distribution $\triangle r^{-k}$. We start letting $r=\left(x_{1}^{2}+\cdots+x_{m}^{2}\right)^{1 / 2}$ and consider the functional $\Delta r^{-k}$ (where $\triangle$ denotes the Laplacian) defined by

$$
\left(\triangle r^{-k}, \phi\right)=\left(r^{-k}, \triangle \phi\right)=\int_{R^{m}} r^{-k} \triangle \phi(x) d x,
$$

where $k<m$ and $\phi(x) \in \mathscr{D}_{m}$, the space of infinitely differentiable functions of the variable $x=\left(x_{1}, x_{2}, \ldots, x_{m}\right)$ with compact support. Because $r^{-k}$ is locally summable, $\triangle r^{-k}$ is a regular distribution.

By Green's theorem for $m \geq 3$, it particularly follows from [5] that

$$
\frac{1}{(2-m) \Omega_{m}} \Delta r^{2-m}=\delta(x),
$$

where $\Omega_{m}=2 \pi^{m / 2} / \Gamma(m / 2)$ is the hypersurface area of the unit sphere imbedded in Euclidean space of $m$-dimension.

A similar calculation for dimension $m=2$ leads to the result

$$
\frac{1}{2 \pi} \triangle \ln r=\delta(x) \text {. }
$$


On making substitution of spherical coordinates in (1.1), we come to

$$
\left(\triangle r^{-k}, \phi\right)=\int_{0}^{\infty} r^{-k}\left\{\int_{r=1} \triangle \phi(\omega) d \omega\right\} r^{m-1} d r,
$$

where $d \omega$ is the hypersurface element on the unit sphere. The integral appearing in the above integrand can be written in the form

$$
\int_{r=1} \triangle \phi(\omega) d \omega=\Omega_{m} S_{\triangle \phi}(r),
$$

where $S_{\triangle \phi}(r)$ is the mean value of $\triangle \phi$ on the sphere of radius $r$.

It could be proved by the well-known Pizetti's formula in [4] that $S_{\triangle \phi}(r)$ is infinitely differentiable for $r \geq 0$, has bounded support, and that

$$
\begin{aligned}
S_{\triangle \phi}(r) & =\triangle \phi(0)+\frac{1}{2 !} S_{\triangle \phi}^{\prime \prime}(0) r^{2}+\cdots+\frac{1}{(2 k) !} S_{\triangle \phi}^{(2 k)}(0) r^{2 k}+\cdots \\
& =\sum_{k=0}^{\infty} \frac{\triangle^{k+1} \phi(0) r^{2 k}}{2^{k} k ! m(m+2) \cdots(m+2 k-2)} .
\end{aligned}
$$

Note that when $k=0$, the first term of the above series is defined as $\triangle \phi(0)$.

From (1.4) and (1.5), we obtain

$$
\begin{aligned}
\left(\triangle r^{-k}, \phi\right) & =\Omega_{m} \int_{0}^{\infty} r^{-k+m-1} S_{\triangle \phi}(r) d r \\
& =\Omega_{m} \int_{0}^{1} r^{-k+m-1} S_{\triangle \phi}(r) d r+\Omega_{m} \int_{1}^{\infty} r^{-k+m-1} S_{\triangle \phi}(r) d r \\
& \triangleq I_{1}+I_{2} .
\end{aligned}
$$

It obviously follows that

$$
I_{2}=\Omega_{m} \int_{1}^{\infty} r^{-k+m-1} S_{\triangle \phi}(r) d r
$$

is well defined for any positive integer $k$.

In order to normalize $I_{1}$ for $k \geq m$, we need the following equation (see [6]):

$$
\begin{aligned}
\left(x_{[0,1]}^{-n}, \phi\right)= & \sum_{j=1}^{n-1} \frac{\phi^{(j-1)}(0)}{(j-1) !(-n+j)} \\
& +\int_{0}^{1} x^{-n}\left[\phi(x)-\phi(0)-x \phi^{\prime}(0)-\cdots-\frac{x^{n-1}}{(n-1) !} \phi^{(n-1)}(0)\right] d x,
\end{aligned}
$$

where $n \geq 1$.

Setting $n=1,2$ in (1.9), we have

$$
\begin{aligned}
& \left(x_{[0,1]}^{-1}, \phi\right)=\int_{0}^{1} x^{-1}[\phi(x)-\phi(0)] d x, \\
& \left(x_{[0,1]}^{-2}, \phi\right)=-\phi(0)+\int_{0}^{1} x^{-2}\left[\phi(x)-\phi(0)-x \phi^{\prime}(0)\right] d x,
\end{aligned}
$$

respectively. 
Using (1.9), we normalize $I_{1}$ for $k \geq m$ by

$$
\begin{aligned}
I_{1}=\Omega_{m} \int_{0}^{1} r^{-k+m-1} S_{\triangle \phi}(r) d r & \\
=\Omega_{m} \sum_{j=1}^{k-m} \frac{S_{\triangle \phi}^{(j-1)}(0)}{(j-1) !(-k+m-1+j)} & \\
& \quad+\Omega_{m} \int_{0}^{1} r^{-k+m-1}\left[S_{\triangle \phi}(r)-S_{\triangle \phi}(0)-r S_{\triangle \phi}^{\prime}(0)\right. \\
& \left.\quad \cdots-\frac{r^{k-m}}{(k-m) !} S_{\triangle \phi}^{(k-m)}(0)\right] d r .
\end{aligned}
$$

In particular for $k=m$, we obtain

$$
\left(\triangle r^{-m}, \phi\right)=\Omega_{m} \int_{0}^{1} r^{-1}\left[S_{\triangle \phi}(r)-S_{\triangle \phi}(0)\right] d r+\Omega_{m} \int_{1}^{\infty} r^{-1} S_{\triangle \phi}(r) d r .
$$

2. The product $r^{-k} \cdot \triangle^{l} \delta$. The following $\delta$-sequence was used by Fisher around 1969. Let $\rho(x)$ be a fixed infinitely differentiable function defined on $\mathbb{R}$ with the following properties:

(i) $\rho(x) \geq 0$,

(ii) $\rho(x)=0$ for $|x| \geq 1$,

(iii) $\rho(x)=\rho(-x)$,

(iv) $\int_{-1}^{1} \rho(x) d x=1$.

The function $\delta_{n}(x)$ is defined by $\delta_{n}(x)=n \rho(n x)$ for $n=1,2, \ldots$ It follows that $\left\{\delta_{n}(x)\right\}$ is a regular sequence of infinitely differentiable functions converging to the Dirac delta-function $\delta(x)$.

Now let $\mathscr{D}$ be the space of infinitely differentiable functions of a single variable with compact support and let $\mathscr{D}^{\prime}$ be the space of distributions defined on $\mathscr{D}$. Then if $f$ is an arbitrary distribution in $\mathscr{D}^{\prime}$, we define

$$
f_{n}(x)=\left(f * \delta_{n}\right)(x)=\left(f(t), \delta_{n}(x-t)\right)
$$

for $n=1,2, \ldots$ It follows that $\left\{f_{n}(x)\right\}$ is a regular sequence of infinitely differentiable functions converging to the distribution $f(x)$ in $\mathscr{D}^{\prime}$.

The following definition for the non-commutative neutrix product $f \cdot g$ of two distributions $f$ and $g$ in $\mathscr{D}^{\prime}$ was given by Fisher in [1].

DEFINITION 2.1. Let $f$ and $g$ be distributions in $\mathscr{D}^{\prime}$ and let $g_{n}=g * \delta_{n}$. We say that the neutrix product $f \cdot g$ of $f$ and $g$ exists and is equal to $h$ if

$$
N-\lim _{n \rightarrow \infty}\left(f g_{n}, \phi\right)=(h, \phi)
$$

for all functions $\phi$ in $\mathscr{D}$, where $N$ is the neutrix (see [9]) having domain $N^{\prime}=\{1,2, \ldots\}$ and range $N^{\prime \prime}$, the real numbers, with negligible functions that are finite linear sums of the functions

$$
n^{\lambda} \ln ^{r-1} n, \quad \ln ^{r} n \quad(\lambda>0, r=1,2, \ldots)
$$


and all functions of $n$ which converge to zero in the normal sense as $n$ tends to infinity.

With Definition 2.1, a lot of products of distributions have been computed by Fisher and Al-Sirehy (see [1, 2]), such as

$$
\begin{gathered}
x_{+}^{\lambda} \cdot x_{-}^{-\lambda-r}=(-1)^{r-1} x_{-}^{\lambda} \cdot x_{+}^{-\lambda-r}=-\frac{\pi \csc (\pi \lambda)}{2(r-1) !} \delta^{(r-1)}(x), \\
(x+i 0)^{\lambda} \cdot(x+i 0)^{-\lambda-r}=x^{-r}+\frac{(-1)^{r} i \pi}{(r-1) !} \delta^{(r-1)}(x)=(x+i 0)^{-r}, \\
|x|^{\lambda} \cdot|x|^{-\lambda-2 r}=x^{-2 r},
\end{gathered}
$$

for a noninteger $\lambda$ and $r=1,2, \ldots$.

REMARK 2.2. The product of Definition 2.1 is not symmetric and hence $f \cdot g \neq g \cdot f$ in general. Furthermore, it is nonassociative since

$$
\left(x^{-1} \cdot x\right) \cdot \delta(x)=1 \cdot \delta(x)=\delta(x) \neq 0=x^{-1} \cdot 0=x^{-1} \cdot(x \cdot \delta(x))
$$

In order to give a definition for a neutrix product $f \cdot g$ of two distributions in $\mathscr{D}_{m}^{\prime}$, the space of distributions defined $\mathscr{D}_{m}$. We may attempt to define a $\delta$-sequence in $\mathscr{D}_{m}$ by simply putting (see [3])

$$
\delta_{n}\left(x_{1}, \ldots, x_{m}\right)=\delta_{n}\left(x_{1}\right) \cdots \delta_{n}\left(x_{m}\right)
$$

where $\delta_{n}$ is defined as above. However, this definition is very difficult to use for distributions in $\mathscr{D}_{m}^{\prime}$ which are functions of $r$. We therefore consider the following approach (see $[8]$ ).

Let $\rho(s)$ be a fixed infinitely differentiable function defined on $\mathbb{R}^{+}=[0, \infty)$ having the following properties:

(i) $\rho(s) \geq 0$,

(ii) $\rho(s)=0$ for $s \geq 1$,

(iii) $\int_{R^{m}} \delta_{n}(x) d x=1$,

where $\delta_{n}(x)=c_{m} n^{m} \rho\left(n^{2} r^{2}\right)$ and $c_{m}$ is the constant satisfying (iii).

It follows that $\left\{\delta_{n}(x)\right\}$ is a regular $\delta$-sequence of infinitely differentiable functions converging to $\delta(x)$ in $\mathscr{D}_{m}^{\prime}$.

DEFINITION 2.3. Let $f$ and $g$ be distributions in $\mathscr{D}_{m}^{\prime}$ and let

$$
g_{n}(x)=\left(g * \delta_{n}\right)(x)=\left(g(x-t), \delta_{n}(t)\right),
$$

where $t=\left(t_{1}, t_{2}, \ldots, t_{m}\right)$. We say that the neutrix product $f \cdot g$ of $f$ and $g$ exists and is equal to $h$ if

$$
N-\lim _{n \rightarrow \infty}\left(f g_{n}, \phi\right)=(h, \phi),
$$

where $\phi \in \mathscr{D}_{m}$ and the $N$-limit is defined as above. 
By Definition 2.3, Li and Fisher [6, 8] showed that the noncommutative neutrix product $r^{-k} \cdot \triangle^{l} \delta$ exists and

$$
\begin{aligned}
r^{-2 k} \cdot \triangle^{l} \delta & =\frac{l !}{2^{k}(l+k) !(m+2 l)(m+2 l+2) \cdots(m+2 l+2 k-2)} \triangle^{l+k} \delta, \\
r^{1-2 k} \cdot \triangle^{l} \delta & =0
\end{aligned}
$$

where $k$ and $l$ are nonnegative integers.

It immediately follows from $l=0,1$ that

$$
\begin{aligned}
r^{-2 k} \cdot \delta & =\frac{\triangle^{k} \delta}{2^{k} k ! m(m+2) \cdots(m+2 k-2)}, \\
r^{1-2 k} \cdot \delta & =0, \\
r^{-2 k} \cdot \triangle \delta & =\frac{\triangle^{1+k} \delta}{2^{k}(k+1) !(m+2)(m+4) \cdots(m+2 k)}, \\
r^{1-2 k} \cdot \triangle \delta & =0 .
\end{aligned}
$$

3. Main results. The following two lemmas will play an important role in obtaining the product $\Delta r^{-k} \cdot \delta$ as well as others.

LEMMA 3.1. Let $D_{i}=\partial / \partial x_{i}$. Then for $k \geq 0$,

$$
\sum_{i=1}^{m} \triangle^{k}\left(x_{i} D_{i} \phi\right)=2 k \triangle^{k} \phi+\sum_{i=1}^{m} x_{i} D_{i}\left(\triangle^{k} \phi\right),
$$

where $\phi \in \mathscr{D}_{m}$.

Proof. We use an inductive method to show the lemma. It is obviously true for $k=0$. Assuming $k=1$, we have

$$
\triangle\left(x_{i} D_{i} \phi\right)=2 \frac{\partial^{2}}{\partial x_{i}^{2}} \phi+x_{i} D_{i}(\triangle \phi)
$$

simply by calculating the left-hand side. As for the sum,

$$
\sum_{i=1}^{m} \triangle\left(x_{i} D_{i} \phi\right)=2 \triangle \phi+\sum_{i=1}^{m} x_{i} D_{i}(\triangle \phi) .
$$

By the inductive hypothesis, equation (3.1) holds for the case of $k-1$, that is,

$$
\sum_{i=1}^{m} \triangle^{k-1}\left(x_{i} D_{i} \phi\right)=2(k-1) \triangle^{k-1} \phi+\sum_{i=1}^{m} x_{i} D_{i}\left(\triangle^{k-1} \phi\right) .
$$


Hence, it follows that

$$
\begin{aligned}
\sum_{i=1}^{m} \triangle^{k}\left(x_{i} D_{i} \phi\right) & =\triangle \sum_{i=1}^{m} \triangle^{k-1}\left(x_{i} D_{i} \phi\right)=\triangle\left\{2(k-1) \triangle^{k-1} \phi+\sum_{i=1}^{m} x_{i} D_{i}\left(\triangle^{k-1} \phi\right)\right\} \\
& =2(k-1) \triangle^{k} \phi+\sum_{i=1}^{m} \triangle\left(x_{i} D_{i}\left(\triangle^{k-1} \phi\right)\right) \\
& =2(k-1) \triangle^{k} \phi+\sum_{i=1}^{m}\left\{2 \frac{\partial^{2}}{\partial x_{i}^{2}} \triangle^{k-1} \phi+x_{i} D_{i}\left(\triangle^{k} \phi\right)\right\} \\
& =2(k-1) \triangle^{k} \phi+2 \triangle^{k} \phi+\sum_{i=1}^{m} x_{i} D_{i}\left(\triangle^{k} \phi\right) \\
& =2 k \triangle^{k} \phi+\sum_{i=1}^{m} x_{i} D_{i}\left(\triangle^{k} \phi\right) .
\end{aligned}
$$

This completes the proof of Lemma 3.1.

LEMMA 3.2.

$$
\sum_{i=1}^{m} D_{i}\left(x_{i} \triangle^{k} \delta\right)=-2 k \triangle^{k} \delta
$$

where $k \geq 0$.

Proof. Applying (3.1), we have

$$
\begin{aligned}
\left(\sum_{i=1}^{m} D_{i}\left(x_{i} \triangle^{k} \delta\right), \phi\right) & =\left(\delta,-\sum_{i=1}^{m} \triangle^{k}\left(x_{i} D_{i} \phi\right)\right) \\
& =\left(\delta,-\left\{2 k \triangle^{k} \phi+\sum_{i=1}^{m} x_{i} D_{i}\left(\triangle^{k} \phi\right)\right\}\right) \\
& =-2 k \triangle^{k} \phi(0) \\
& =\left(-2 k \triangle^{k} \delta, \phi\right) .
\end{aligned}
$$

Therefore, we have reached our conclusion in Lemma 3.2.

THEOREM 3.3. The noncommutative neutrix product $\triangle r^{-k} \cdot \delta$ exists. Furthermore,

$$
\begin{aligned}
\triangle r^{-2 k} \cdot \delta & =\frac{k(2 k+2-m)}{2^{k}(k+1) ! m(m+2) \cdots(m+2 k)} \triangle^{k+1} \delta, \\
\triangle r^{1-2 k} \cdot \delta & =0
\end{aligned}
$$

where $k$ is any positive integer.

Proof. We note that $r^{-k}$ is a locally summable function on $\mathbb{R}^{m}$ for $k=1,2, \ldots$, $m-1$. With Definition 2.3, we naturally consider

$$
\begin{aligned}
I & =\left(\triangle r^{-k} \cdot \delta_{n}, \phi\right)=\left(r^{-k}, \triangle\left(\delta_{n} \phi\right)\right) \\
& =\left(r^{-k}, \triangle \delta_{n} \phi\right)+\left(r^{-k}, \delta_{n} \triangle \phi\right)+2 \sum_{i=1}^{m}\left(r^{-k}, D_{i} \delta_{n} D_{i} \phi\right) \\
& =I_{1}+I_{2}+I_{3} .
\end{aligned}
$$


Clearly,

$$
\left(r^{-k} \cdot \triangle \delta, \phi\right)=N-\lim _{n \rightarrow \infty} I_{1}=N-\lim _{n \rightarrow \infty}\left(r^{-k}, \triangle \delta_{n} \phi\right)
$$

and using (2.11), we obtain

$$
\begin{aligned}
\left(r^{-2 k} \cdot \triangle \delta, \phi\right) & =\frac{\left(\triangle^{k+1} \delta, \phi\right)}{2^{k}(k+1) !(m+2)(m+4) \cdots(m+2 k)}, \\
\left(r^{1-2 k} \cdot \triangle \delta, \phi\right) & =0,
\end{aligned}
$$

which indeed hold for any positive integer $k$.

It follows from $[6,8]$ that

$$
N-\lim _{n \rightarrow \infty} I_{2}=N-\lim _{n \rightarrow \infty}\left(r^{-k}, \delta_{n} \triangle \phi\right)=\frac{S_{\triangle \phi}^{(k)}(0)}{k !}
$$

and applying Pizetti's formula, we have

$$
\begin{aligned}
N-\lim _{n \rightarrow \infty}\left(r^{-2 k}, \delta_{n} \triangle \phi\right) & =\left(\frac{\triangle^{k+1} \delta}{2^{k} k ! m(m+2) \cdots(m+2 k-2)}, \phi\right), \\
N-\lim _{n \rightarrow \infty}\left(r^{1-2 k}, \delta_{n} \triangle \phi\right) & =(0, \phi),
\end{aligned}
$$

which are again true for any positive integer $k$.

Putting $\psi_{i}=x_{i} D_{i} \phi$, we deduce that

$$
I_{3}=4 c_{m} n^{m+2} \Omega_{m} \sum_{i=1}^{m} \int_{0}^{1 / n} r^{m-k-1} \rho^{\prime}\left(n^{2} r^{2}\right) S_{\psi_{i}}(r) d r
$$

and by Taylor's formula, we obtain

$$
S_{\psi_{i}}(r)=\sum_{j=0}^{k+1} \frac{S_{\psi_{i}}^{(j)}(0)}{j !} r^{j}+\frac{S_{\psi_{i}}^{(k+2)}(0)}{(k+2) !} r^{k+2}+\frac{S_{\psi_{i}}^{(k+3)}(\zeta r)}{(k+3) !} r^{k+3},
$$

where $0<\zeta<1$. Hence

$$
\begin{aligned}
I_{3}= & 4 c_{m} \Omega_{m} n^{m+2} \sum_{i=1}^{m} \sum_{j=0}^{k+1} \frac{S_{\psi_{i}}^{(j)}(0)}{j !} \int_{0}^{1 / n} r^{m-k-1} \rho^{\prime}\left(n^{2} r^{2}\right) r^{j} d r \\
& +4 c_{m} \Omega_{m} n^{m+2} \sum_{i=1}^{m} \int_{0}^{1 / n} r^{m-k-1} \rho^{\prime}\left(n^{2} r^{2}\right) \frac{S_{\psi_{i}}^{(k+2)}(0)}{(k+2) !} r^{k+2} d r \\
& +4 c_{m} \Omega_{m} n^{m+2} \sum_{i=1}^{m} \int_{0}^{1 / n} r^{m-k-1} \rho^{\prime}\left(n^{2} r^{2}\right) \frac{S_{\psi_{i}}^{(k+3)}(\zeta r)}{(k+3) !} r^{k+3} d r \\
= & I_{1}^{\prime}+I_{2}^{\prime}+I_{3}^{\prime},
\end{aligned}
$$

respectively. Employing the substitution $t=n r$, we get

$$
I_{1}^{\prime}=4 c_{m} \Omega_{m} \sum_{i=1}^{m} \sum_{j=0}^{k+1} n^{k+2-j} \frac{S_{\psi_{i}}^{(j)}(0)}{j !} \int_{0}^{1} t^{m+j-k-1} \rho^{\prime}\left(t^{2}\right) d t
$$


whence

$$
N-\lim _{n \rightarrow \infty} I_{1}^{\prime}=0
$$

as for

$$
I_{2}^{\prime}=4 c_{m} \Omega_{m} \sum_{i=1}^{m} \frac{S_{\psi_{i}}^{(k+2)}(0)}{(k+2) !} \int_{0}^{1} t^{m+1} \rho^{\prime}\left(t^{2}\right) d t
$$

integrating by parts, we have

$$
\begin{aligned}
4 c_{m} \Omega_{m} \int_{0}^{1} t^{m+1} \rho^{\prime}\left(t^{2}\right) d t & =2 c_{m} \Omega_{m} \int_{0}^{1} t^{m} d \rho\left(t^{2}\right) \\
& =-2 c_{m} \Omega_{m} \cdot m \int_{0}^{1} t^{m-1} \rho\left(t^{2}\right) d t \\
& =-2 m \int_{R^{m}} \delta_{n}(x) d x=-2 m .
\end{aligned}
$$

Hence

$$
I_{2}^{\prime}=-2 m \sum_{i=1}^{m} \frac{S_{\psi_{i}}^{(k+2)}(0)}{(k+2) !}=-\frac{2 m}{(k+2) !} \sum_{i=1}^{m} S_{\psi_{i}}^{(k+2)}(0) .
$$

Putting

$$
M=\sup \left\{\left|S_{\psi_{i}}^{(k+3)}(r)\right|: r \in \mathbb{R}^{+} \text {and } 1 \leq i \leq m\right\}
$$

we obtain

$$
\left|I_{3}^{\prime}\right| \leq 4 c_{m} \Omega_{m} \frac{m M}{n(k+3) !} \int_{0}^{1} t^{m+2}\left|\rho^{\prime}\left(t^{2}\right)\right| d t \rightarrow 0 \quad \text { as } n \rightarrow \infty .
$$

Hence it follows from above that

$$
N-\lim _{n \rightarrow \infty} I_{3}=I_{2}^{\prime}=-\frac{2 m}{(k+2) !} \sum_{i=1}^{m} S_{\psi_{i}}^{(k+2)}(0)
$$

which can be extended to the case $k \geq m$ by utilizing the normalization procedure of $\mu(x) x_{+}^{\lambda}$ (see [7]) and (1.11) in Section 1 .

On using Pizetti's formula and Lemma 3.2, we come to

$$
\begin{aligned}
-\frac{2 m}{(2 k+2) !} \sum_{i=1}^{m} S_{\psi_{i}}^{(2 k+2)}(0) & =\frac{-2 m \sum_{i=1}^{m} \triangle^{k+1} \psi_{i}(0)}{2^{k+1}(k+1) ! m(m+2) \cdots(m+2 k)} \\
& =\frac{2 m \sum_{i=1}^{m}\left(D_{i}\left(x_{i} \triangle^{k+1} \delta\right), \phi\right)}{2^{k+1}(k+1) ! m(m+2) \cdots(m+2 k)} \\
& =\frac{-4 m(k+1)\left(\triangle^{k+1} \delta, \phi\right)}{2^{k+1}(k+1) ! m(m+2) \cdots(m+2 k)}
\end{aligned}
$$

by substituting $\psi_{i}=x_{i} D_{i} \phi$ back and obviously

$$
-\frac{2 m}{(2 k+1) !} \sum_{i=1}^{m} S_{\psi_{i}}^{(2 k+1)}(0)=0 .
$$

Therefore Theorem 3.3 follows from (3.11), (3.13), (3.25), and (3.26). 
In particular for $k=1,2$, we have the following:

$$
\begin{aligned}
& \triangle r^{-2} \cdot \delta=\frac{4-m}{4 m(m+2)} \triangle^{2} \delta, \quad \triangle \frac{1}{r} \cdot \delta=0, \\
& \triangle r^{-4} \cdot \delta=\frac{(6-m)}{12 m(m+2)(m+4)} \triangle^{3} \delta, \quad \triangle \frac{1}{r^{3}} \cdot \delta=0,
\end{aligned}
$$

where $m$ is the dimension.

ACKNOWLEDGEMENT. This research was supported by National Science and Engineering Research Council (NSERC) and Augustana research grant.

\section{REFERENCES}

[1] B. Fisher, On defining the convolution of distributions, Math. Nachr. 106 (1982), 261-269. MR 83m:46066. Zbl 0499.46024.

[2] B. Fisher and F. Al-Sirehy, On the non-commutative neutrix product of $x_{+}^{\lambda}$ and $x_{+}^{-\lambda-r}$, Publ. Inst. Math. (Beograd) (N.S.) 65(79) (1999), 112-122. MR 2000k:46047. Zbl 0952.46023.

[3] B. Fisher and C. K. Li, On defining a noncommutative product of distributions in $m$ variables, J. Natur. Sci. Math. 31 (1991), no. 2, 95-102. MR 93a:46073. Zbl 0772.46016.

[4] I. M. Gel'fand and G. E. Shilov, Generalized Functions. Vol. I: Properties and Operations, Academic Press, New York, 1964. MR 29\#3869. Zbl 0115.33101.

[5] C. K. Li, A note on the product $r^{-k} \cdot \nabla\left(\Delta r^{2-m}\right)$, to appear in Integral Transform. Spec. Funct.

[6] _ A new product of distributions in $m$ variables, The Proceedings of the International Conference on Mathematics and Its Applications in the New Millennium (Department of Mathematics, University of Putra, Malaysia, July 18-21), 2000.

[7] The product of $r^{-k}$ and $\nabla \delta$ on $\mathbf{R}^{m}$, Int. J. Math. Math. Sci. 24 (2000), no. 6, 361-369. MR 2001i:46068. Zbl 0963.46020.

[8] C. K. Li and B. Fisher, Example of the neutrix product of distributions on $\mathbf{R}^{m}$, Rad. Mat. 6 (1990), no. 1, 129-137. MR 91m:46055. Zbl 0735.46029.

[9] J. G. van der Corput, Introduction to the neutrix calculus, J. Analyse Math. 7 (1959/1960), 281-399. MR 23\#A1989. Zbl 0097.10503.

C. K. Li: Department of Mathematical Sciences, Augustana University College, CAmrose, Alberta, CANADA T4V 2R3

E-mail address: $1 \mathrm{i} @ @ a u g u s t a n a . c a$ 


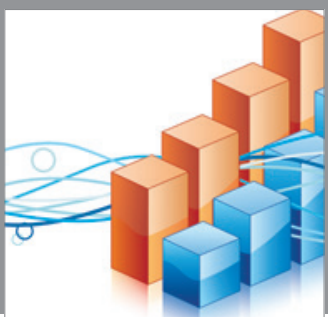

Advances in

Operations Research

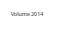

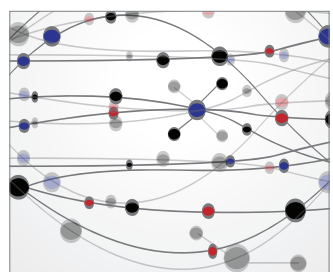

\section{The Scientific} World Journal
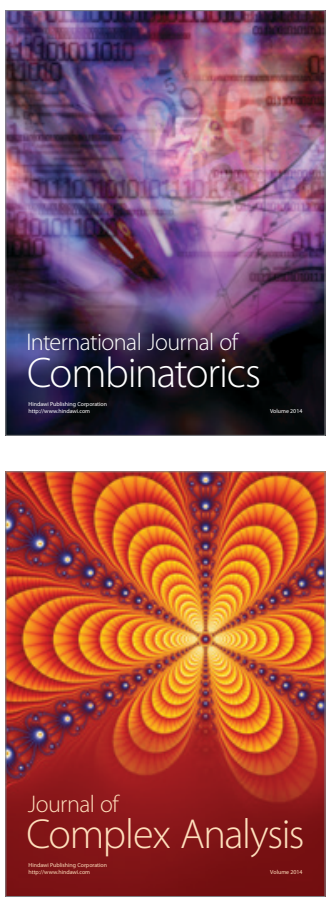

International Journal of

Mathematics and

Mathematical

Sciences
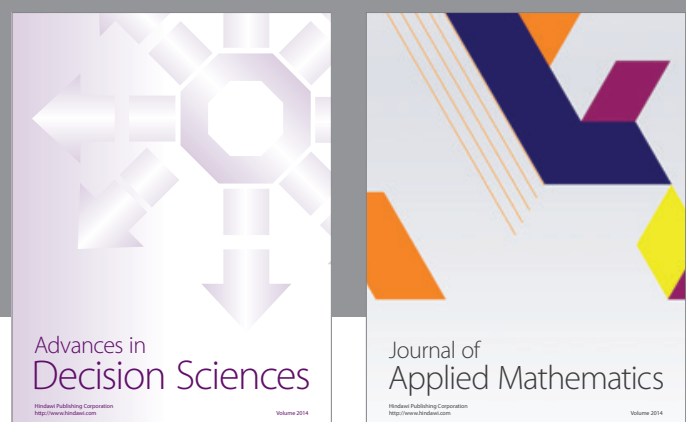

Journal of

Applied Mathematics


Submit your manuscripts at http://www.hindawi.com
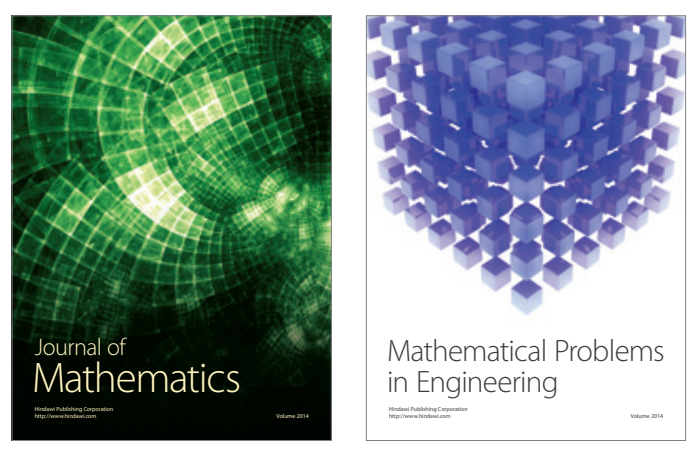

Mathematical Problems in Engineering
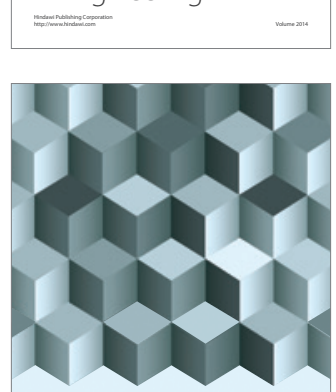

Journal of

Function Spaces
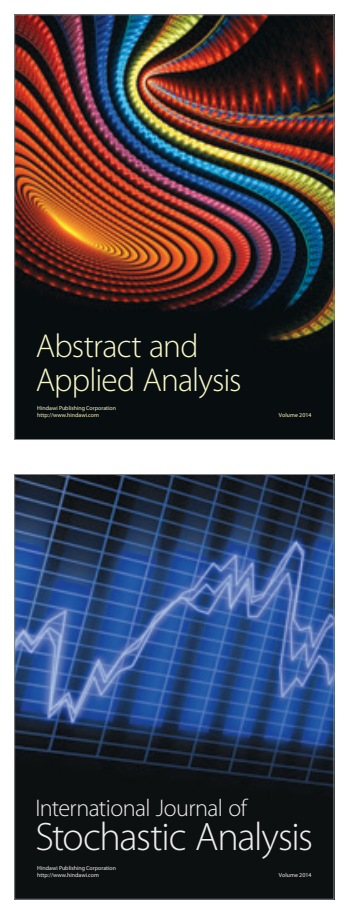

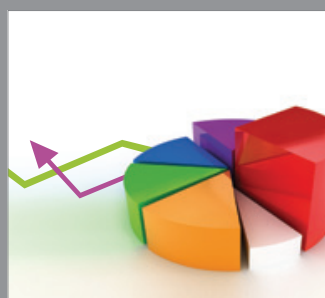

ournal of

Probability and Statistics

Promensencen
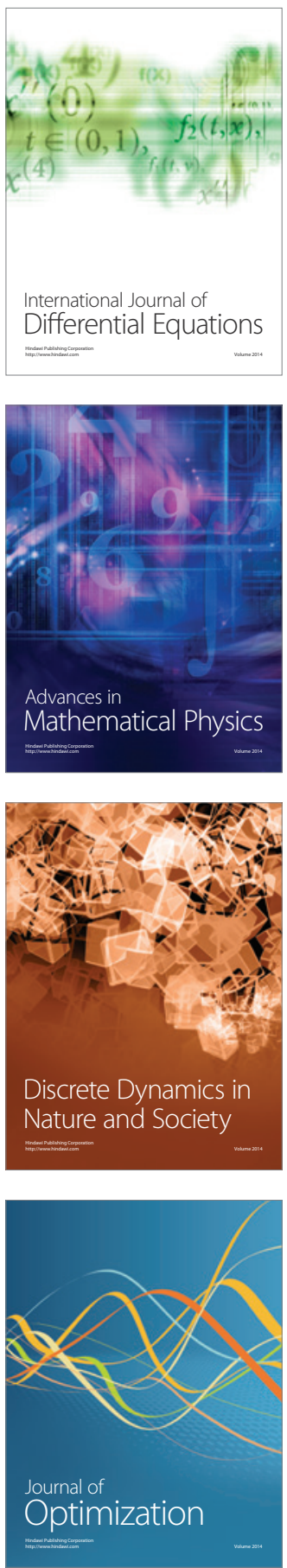